\title{
Biliary Granular Cell Tumor
}

\author{
Changwon Jung · Ilyeong Heo • Sang Bum Kim ${ }^{1}$ - Sunhoo Park • Soo Youn Cho \\ Departments of Pathology and 'Surgery, Korea Cancer Center Hospital, Korea Institute of Radiological and Medical Sciences, Seoul, Korea
}

Granular cell tumor (GCT) is a benign neoplasm showing neuroectodermal differentiation and is most commonly found in the head and neck region, including the tongue. ${ }^{1}$ This tumor is now believed to occur in virtually any site of the body, including skin, breast, and gastrointestinal tract, with less than $1 \%$ developing in the biliary tract. ${ }^{2}$ To our knowledge, only one case of GCT of the biliary tract has been reported in Korea, which occurred in the gallbladder. ${ }^{3}$ Herein, we present another case of GCT of the biliary tract.

\section{CASE REPORT}

A 62-year-old female was referred to our hospital for management of gastric adenocarcinoma that had been found during a periodic health examination. She had been receiving treatment for hypertension and diabetes mellitus for 20 years. Abdominal computed tomography for a diagnostic workup revealed focal dilatation of the right posterior hepatic duct, and subsequent imaging with magnetic resonance imaging and magnetic resonance cholangiopancreatography showed a stricture at the right hepatic duct (Fig. 1A). Laboratory findings were unremarkable.

Under the presumptive diagnosis of synchronous cholangiocarcinoma and gastric carcinoma, she underwent hepatic right lobectomy with radical subtotal gastrectomy. On a resected specimen, a $0.9 \times 0.4 \mathrm{~cm}$ sized, ill-defined, nonencapsulated tumor was noted in the extrahepatic portion of the right hepatic duct (Fig. 1B). The bile duct mucosa was grossly intact, and the tumor was located in the submucosal layer. The cut surface of the

\section{Corresponding Author}

Soo Youn Cho, M.D.

Department of Pathology, Korea Cancer Center Hospital, Korea Institute of Radiological and Medical Sciences, 75 Nowon-ro, Nowon-gu, Seoul 139-706, Korea Tel: +82-2-970-2545, Fax: +82-2-970-2430, E-mail: pathsycho@naver.com

Received: August 31, 2014 Revised: October 4, 2014

Accepted: October 7, 2014 tumor was gray to whitish, solid, firm, and infiltrative (Fig. 1C). The margins were clear on frozen biopsy. On microscopic examination, the tumor was composed of large polygonal cells with abundant eosinophilic granular cytoplasm, and the nuclei were small, dark, uniform, and centrally located (Fig. 2A, B). The overlying mucosa was atrophic and showed autolysis (Fig. 2A). The tumor cells were diffusely positive for periodic acid-Schiff (PAS), CD68, and S100 protein (Fig. 2C). A diagnosis of GCT was made. The stomach tumor was papillary adenocarcinoma (pT1N0Mx). The patient remains healthy at 20 months after the resection, without any signs of complication.

\section{DISCUSSION}

The first case of GCT was reported by Abrikossoff in 1926 in the skeletal muscle of the tongue. ${ }^{4}$ Since then, there have been some discrepancies regarding the origin of GCT based on histologic and immunohistochemical findings, including myogenic, histiogenic, neurogenic, and multicentric histogeneses. ${ }^{5}$ The exact histogenesis is still unclear, but a neural origin, more specifically Schwannian type neuroectodermal origin, is favored by many authors ${ }^{1,5,6}$ because the tumor cells show positivity for S100 protein, which is normally found in the central nervous system and peripherally in Schwann cells. ${ }^{6,7}$

GCT has a distinct histological appearance, being composed of polygonal eosinophilic cells that contain cytoplasmic granules strongly reactive to PAS. The cells also contain small, central, and vesicular nuclei, appear as clusters or sheets, and infiltrate diffusely within the surrounding structures. They commonly show perineural infiltration that might lead to local recurrence after incomplete excision. Immunohistochemically, these tumor cells show positivity for $\mathrm{S} 100$ protein, neuron-specific enolase, vimentin, and various other Schwann-cell-related antigens. Mitosis and necrosis are rarely noted. There have been some 

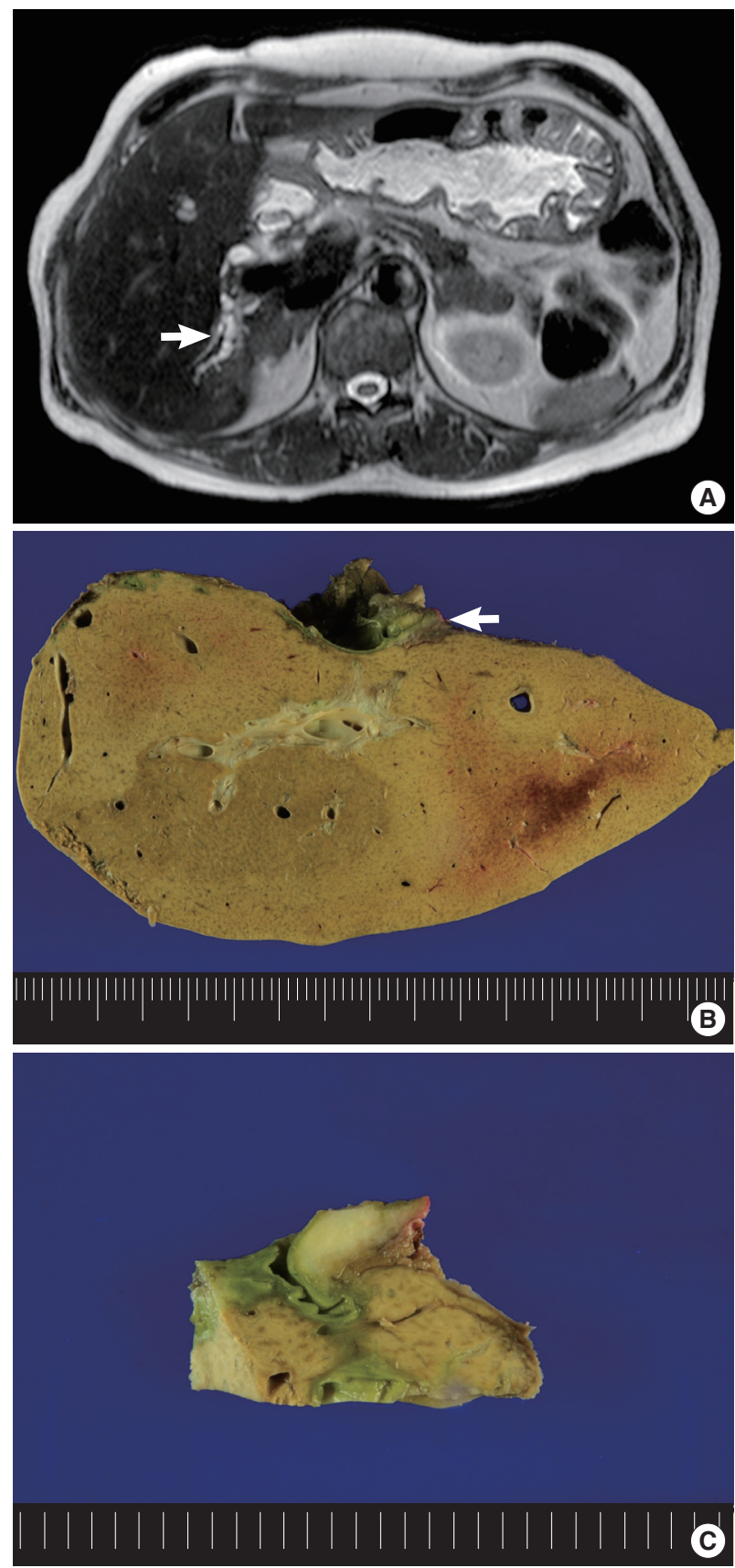

Fig. 1. (A) Magnetic resonance imaging shows focal dilation of the intrahepatic bile duct in the right posterior segment (arrow) with atrophy. (B) The specimen shows a small tumor in the extrahepatic portion of the right hepatic duct (arrow) and dilated intrahepatic bile ducts. (C) A whitish infiltrative tumor is noted in the submucosal layer of the bile duct.

reports of malignant GCT; however, there is no report of malignant biliary tract GCT to date.

GCT can develop at any age but is more prevalent in the fifth and sixth decades and shows a slight male predominance. It is
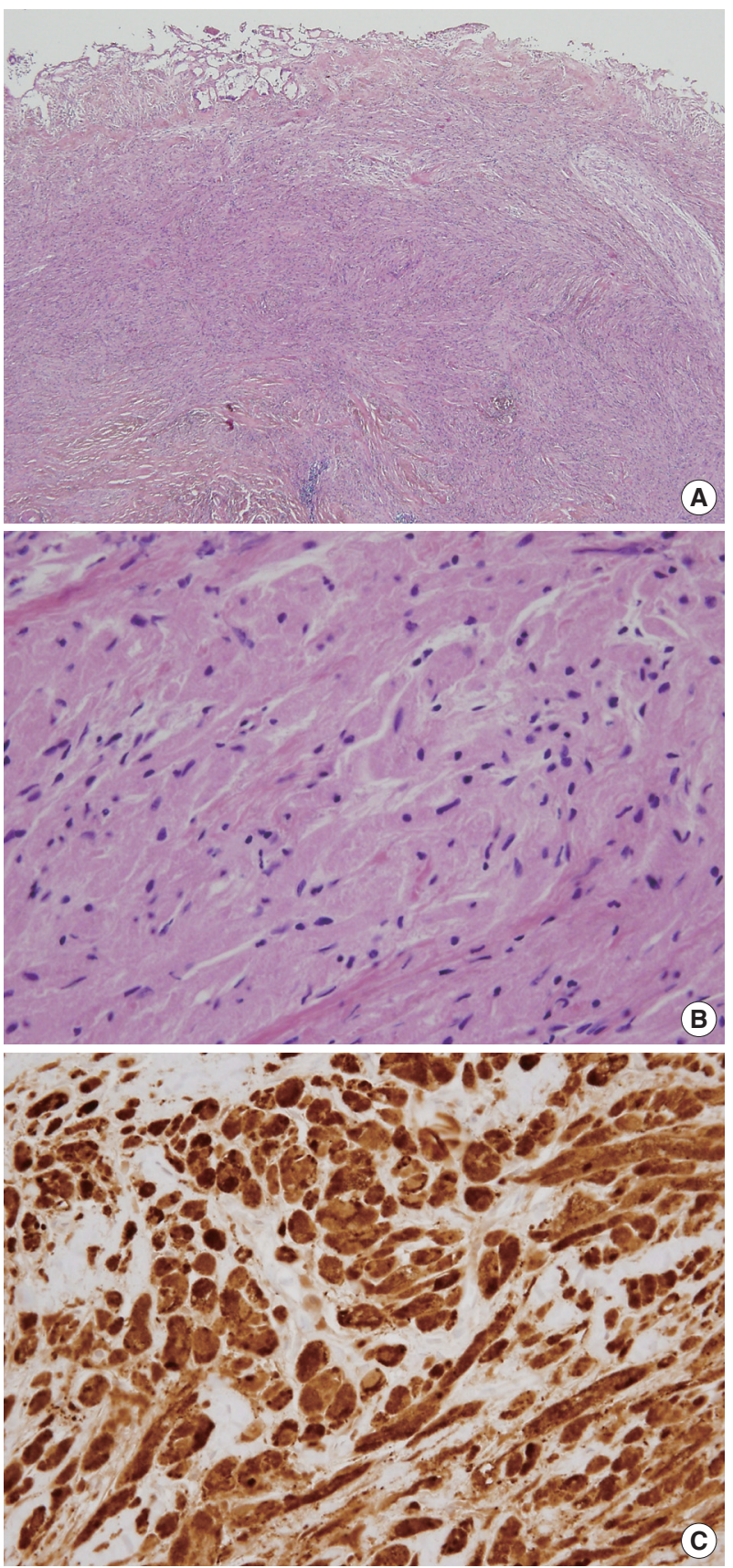

Fig. 2. Histologic findings of the tumor. (A) Diffuse, infiltrative tumor is noted in the submucosa of the bile duct. (B) The large tumor cells show eosinophilic granular cytoplasm. (C) The tumor cells are strongly positive for S100 protein.

typically solitary and smaller than $3 \mathrm{~cm}$ in diameter. GCT has been found in almost every part of the body, with the head and neck region being the most commonly affected site, accounting for $45 \%-65 \%$ of cases. ${ }^{1}$

GCT of the biliary tract is very rare, with the first case reported by Coggins in 1952 during autopsy. ${ }^{8}$ Since then, 81 cas- 
es of biliary tract GCT have been reported in the English literature, with only one case reported in a Korean patient. Among reported cases, including our case $(n=82)$, about $52 \%(n=43)$ of the biliary tract GCT has been observed in black women with a median age of 34 years (range, 14 to 91 years). Interestingly, GCT of the biliary tract is more prevalent in slightly younger age and females (female to male ratio, 5.3:1). The younger age at presentation might reflect a spatial problem due to a narrow lumen of the biliary tract. In the literature review, most patients have complained of jaundice (44.4\%), abdominal pain (34.6\%), or both $(11.1 \%))^{7,9,10}$ There have been two cases that required liver transplantation due to secondary biliary cirrhosis. In our case, the tumor was incidentally detected during the stage workup for gastric cancer.

Many patients are clinically and radiologically suspected for cholangiocarcinoma preoperatively; thus, they tend to undergo extensive procedures such as Whipple's operation. However, GCT is almost always benign and can usually be cured by complete excision alone, which is associated with a generally good prognosis. Biliary tract GCT can also be treated with surgical excision with tumor-free margins followed by hepaticojejunostomy.

In summary, biliary GCT can cause symptoms related to biliary obstruction and might present with a clinical impression of cholangiocarcinoma. Thus, GCT should be included in the differential diagnosis of biliary tract tumors, even though the incidence is extremely low.

\section{Conflicts of Interest}

No potential conflict of interest relevant to this article was reported.

\section{REFERENCES}

1. Fletcher CD, Bridge JA, Hogendoorn P, Mertens F. WHO classification of tumours of soft tissue and bone. 4th ed. Lyon: IARC Press, 2013.

2. Karakozis S, Gongora E, Zapas JL, He P, Krishnan J, Kirkpatrick JR. Granular cell tumors of the biliary tree. Surgery 2000; 128: 113-5.

3. Kim DK, Jung YK, Chung DH, et al. Granular cell tumor originating from gallbladder. Korean J Med 2012; 83: 624-8.

4. Abrikossoff A. About myomas originating from striated musculature. Virchows Arch A Pathol Anat 1926; 260: 215-33.

5. Caputo R, Bellone AG, Tagliavini R. Ultrastructure of the granular cell myoblastoma: so-called Abrikossoff's tumor. Arch Dermatol Forsch 1972; 242: 127-36.

6. Le BH, Boyer PJ, Lewis JE, Kapadia SB. Granular cell tumor: immunohistochemical assessment of inhibin-alpha, protein gene product 9.5, S100 protein, CD68, and Ki-67 proliferative index with clinical correlation. Arch Pathol Lab Med 2004; 128: 771-5.

7. Patel AJ, Jakate SM. Granular cell tumor of the biliary tract. Gastroenterol Hepatol (N Y) 2010; 6: 331-6.

8. Coggins RP. Granular-cell myoblastoma of common bile duct: report of a case with autopsy findings. AMA Arch Pathol 1952; 54: 398-402.

9. Bilanović D, Boricić I, Zdravković D, Randjelović T, Stanisavljević N, Toković B. Granular cell tumor of the common hepatic duct presenting as cholangiocarcinoma and acute acalculous cholecystitis. Acta Chir Iugosl 2008; 55: 99-101.

10. Saito J, Kitagawa M, Kusanagi $\mathrm{H}$, et al. Granular cell tumor of the common bile duct: a Japanese case. World J Gastroenterol 2012; 18: $6324-7$ 